\title{
Cyclosporin Treatment in Steroid-resistant and Acutely Exacerbated Interstitial Pneumonia
}

\author{
Sakae Homma, Susumu SaKamoto, Masateru KawabAta, Kazuma Kishi, \\ Eiyasu TsuboI, Noriko MotoI* and Kunihiko Yoshimura
}

\begin{abstract}
Objective The aim of this study was to evaluate the efficacy of cyclosporin A (CsA) in patients with interstitial pneumonia (IP).

Design Retrospective comparative study.

Patients We reviewed 33 patients ( 23 males and 10 females with a mean age of 62.5 years) with histologicallyproven progressive IP who were treated with CsA. All patients had corticosteroid-resistant IP or developed acute exacerbation of IP in their courses.

Results The underlying systemic diseases were: idiopathic interstitial pneumonias (IIPs) in 19 patients, and collagen vascular diseases (CVDs) in 14. The histopathological patterns and underlying diseases of IP were classified as usual interstitial pneumonia (UIP)/idiopathic pulmonary fibrosis (IPF) in 10 patients, cellular-nonspecific interstitial pneumonia (NSIP)/IIPs in 3, fibrotic-NSIP/ IIPs in 5, organizing pneumonia (OP)/IIP in 1, UIP/CVDs in 4, cellular-NSIP/CVDs in 7, fibrotic-NSIP/CVDs in 2, and diffuse alveolar damage (DAD)/CVD in 1, respectively. They received a low dosage of CsA combined with corticosteroids. The prognoses after treatment with CsA were well correlated with histopathological patterns. Cellular-NSIP and OP showed better prognoses than fibrotic-NSIP, UIP or DAD. In addition, CVDs had better prognoses than IIPs, when compared on the basis of the same histopathological patterns. Furthermore, the prognoses in the CsA-treated group were significantly better than in those without CsA treatment in regard to acute exacerbation of UIP/IPF.

Conclusions CsA combined with corticosteroids may be an efficacious treatment for corticosteroid-resistant IP and for acute exacerbation of IPF.

(Internal Medicine 44: 1144-1150, 2005)
\end{abstract}

Key words: idiopathic interstitial pneumonia, idiopathic pulmonary fibrosis, interstitial pneumonia, treatment, cyclosporin, survival rates

\section{Introduction}

Recently, cyclosporin A (CsA) has been shown to be efficacious in the treatment of interstitial pneumonia (IP) associated with polymyositis and dermatomyositis (PM/DM), the conditions of which are refractory to corticosteroid therapy (1-3). However, the efficacy of CsA in idiopathic interstitial pneumonias (IIPs) is still undetermined. CsA primarily inhibits activation of T lymphocytes, by blocking expression of the genes, such as IL-2, which are regulated by the nuclear factor of activated T cells (NF-AT) transcription factor. In addition, it has been reported that CsA exerts direct effects on macrophages. As T lymphocytes and alveolar macrophages play an important role in the pathogenesis of idiopathic pulmonary fibrosis (IPF), CsA may have the potential efficacy as a modulator of the clinical course of IPF. In contrast, suppression of IL-2 by corticosteroid treatment requires interaction with the AP-1 (Fos/Jun) site. These findings suggest that these two agents contribute to the suppression of IL-2 gene expression in an independent as well as additional or synergistic fasion $(4,5)$.

With this background, the present study was aimed at assessing the prognoses of patients with histologically-proven and corticosteroid-resistant progressive interstitial pneumonia who received the treatment with CsA.

\section{Subjects and Methods}

Thirty-three patients (23 males and 10 females) with a mean age of 62.5 years, who were clinically diagnosed as having corticosteroid-resistant IP, or had developed acute ex-

From the Department of Respiratory Medicine, Respiratory Center, Tokyo and *the Department of Pathology, Toranomon Hospital, Tokyo Received for publication March 14, 2005; Accepted for publication July 22, 2005

Reprint requests should be addressed to Dr. Sakae Homma, the Department of Respiratory Medicine, Respiratory Center, Toranomon Hospital, 2-2-2 Toranomon, Minato-ku, Tokyo 105-8470 
acerbation of IP during their illness, were recruited during the period from 1997 through 2004. Corticosteroid-resistant IP was defined as the disease that progresses despite the administration of a minimum dosage of $20 \mathrm{mg} /$ day of prednisolone for more than 12 weeks. All patients received a low dosage (50-200 mg/day) of CsA combined with corticosteroids. Clinical features, chest high-resolution CT (HRCT) scan images, pulmonary function tests, pathological findings, and prognoses of all patients were retrospectively evaluated.

\section{Clinical features}

Clinical symptoms, the nature of the treatments, and prognoses were evaluated. IIPs were diagnosed based on the definition by the ATS/ERS International Consensus Classification of IIPs (6). Primary Sjögren's syndrome ( $\mathrm{SjS}$ ) was diagnosed based on the preliminary criteria for the classification of $\mathrm{SjS}$ established by the European Community (7). $\mathrm{PM} / \mathrm{DM}$ was diagnosed based on the criteria of Bohan and Peter (8). Microscopic polyangiitis (MPA) was diagnosed based on the Chapel Hill Consensus Conference (9). Rheumatoid arthritis (RA) was diagnosed based on the American Rheumatism Association 1987 revised criteria for the classification of rheumatoid arthritis (10).

\section{CT scan images}

Chest HRCT scan images were evaluated before and after treatment by using a High Speed Advantage scanner (GE Medical Systems, Milwaukee, WI, USA). Routine scanning of the entire lung was carried out with sections 10-mm thick. Additional thin-section CT with $1.0 \mathrm{~mm}$-section thickness of the parenchymal abnormalities was performed for all patients. Thin-section CT images were reconstructed with a high-spatial-frequency algorithm and were printed with fixed window settings (lung center, $-500 \mathrm{HU}$ and width, 1,800 $\mathrm{HU})$. A consensus reading of the CT images was conducted by two observers.

\section{Pulmonary function tests}

Lung volume, forced expiratory volume in one second $\left(\mathrm{FEV}_{1.0}\right)$, carbon monoxide diffusing capacity (DLco) and arterial blood gases were measured according to the standard methods with a Chestac-55V (Chest Co. Ltd., Tokyo, Japan) and an ABL510 (Radiometer Co. Ltd., Copenhagen, Denmark).

\section{Morphological analysis}

The histopathological characteristics of IP were analyzed in all patients. Out of these 33 patients, 18 patients underwent lung biopsy by video-assisted thoracoscopic surgery (VATS), and 15 underwent postmortem examinations. The lung specimens were fixed with $10 \%$ formaldehyde and embedded in paraffin, from which 3- $\mu \mathrm{m}$-thick sections were cut and stained with hematoxylin-eosin and elastica van Gieson. The sections were mounted in an aqueous mounting medium and observed by light microscopy to determine the characteristics of the IP. The histopathological patterns of IP based on the definition of the ATS/ERS International Consensus Classification of IIPs (6) were diagnosed by two pathologists.

\section{Survival rates}

The survival rates after treatment with CsA for each histopathological pattern/underlying systemic disease of IP were compared by the log rank test and Kaplan-Meier survival curves were plotted. Furthermore, the survival rates after the onset of acute exacerbation of IPF were compared between the CsA-treated group (9 patients; 9 males with a mean age of 69 years) and the non-CsA-treated group (35 patients; 31 males $/ 4$ females with a mean age of 67 years. IPF was diagnosed by using the then clinical diagnostic criteria established with the aid of the Ministry of Health and Welfare of Japan; 30 patients underwent postmortem examinations, one patient underwent transbronchial lung biopsy and 4 patients had no histological confirmation) in our institution by using the same analytic methods $(11,12)$.

\section{Definition of acute exacerbation of IP}

Acute exacerbation of IP was defined if all the conditions stated below were met. 1) Exacerbation of dyspnea within one month. 2) Newly developed diffuse parenchymal opacities on chest CT and/or chest X-ray. 3) A decrease in $\mathrm{PaO}_{2}$ of more than $10 \mathrm{mmHg}$ from the previous level at rest. 4) An absence of lung infection or heart failure (13).

\section{Criteria for assessment of the responses to the ther- apy for IP}

Based on the criteria of the ATS/ERS International Consensus Statement on IPF (14), the responses to treatment for IP were defined as described below. Improved response to therapy was defined as two or more of the following: 1) An improvement of symptoms, especially an increase in the exercise capability up to the patient's intolerance level caused by breathlessness, or frequent and severe cough and/or sputum. 2) Reduction of parenchymal abnormalities as revealed on chest HRCT scan. 3) Physiological improvement defined by one or more of the following: a) a greater than $10 \%$ increase in forced vital capacity (FVC) or at least $>200$ $\mathrm{ml}$ change, b) a $15 \%$ increase in single-breath DLco or at least $>3 \mathrm{ml} / \mathrm{min} / \mathrm{mmHg}$, c) a greater than $10 \mathrm{mmHg}$ increase in resting $\mathrm{PaO}_{2}$ from the previous level.

Unchanged response to therapy was defined as two of the following: 1) Persistence of parenchymal abnormalities as shown on chest HRCT scan. 2) Physiological stability defined by one or more of the following: a) a change in FVC not greater than $10 \%$, or a $200 \mathrm{ml}$ change, b) a change in single-breath DLco of less than $15 \%$ or $<3 \mathrm{ml} / \mathrm{min} / \mathrm{mmHg}$, c) an increase in resting $\mathrm{PaO}_{2}$ of less than $10 \mathrm{mmHg}$ from the previous level.

A failure to respond to therapy was defined as two or more of the following: 1) A worsening of symptoms, especially dyspnea, or cough and/or sputum. 2) An increase in opacities as revealed on chest HRCT scan. 3) Physiological 
Homma et al

Table 1. Clinical and pathological features

\begin{tabular}{|c|c|c|c|c|}
\hline & UIP/IPF & Non-UIP/IIPs & UIP/CVDs & Non-UIP/CVDs \\
\hline Number of patients & 10 & 9 & 4 & 10 \\
\hline Age $($ mean \pm SD), yr & $69.6 \pm 6.2$ & $62.3 \pm 14.0$ & $67.0 \pm 14.0$ & $53.7 \pm 12.9$ \\
\hline Gender (Male $\cdot$ Female) & $10 \cdot 0$ & $6 \cdot 3$ & $2 \cdot 2$ & $5 \cdot 5$ \\
\hline \multicolumn{5}{|l|}{ Smoking status } \\
\hline Never & 3 & 6 & 3 & 8 \\
\hline Former & 2 & 1 & 0 & 0 \\
\hline Current & 5 & 2 & 1 & 2 \\
\hline \multirow[t]{4}{*}{ Underlying systemic diseases } & None & None & RA: 2 & DM: 7 \\
\hline & & & SjS: 2 & RA: 1 \\
\hline & & & & SjS: 1 \\
\hline & & & & MPA: 1 \\
\hline \multirow[t]{3}{*}{ Histopathological classification of IP } & UIP: 10 & C-NSIP: 3 & UIP: 4 & C-NSIP: 7 \\
\hline & & F-NSIP: 5 & & F-NSIP: 2 \\
\hline & & OP: 1 & & DAD: 1 \\
\hline \multicolumn{5}{|l|}{ Baseline data $($ mean $\pm S D)$} \\
\hline $\mathrm{PaO}_{2} / \mathrm{FiO}_{2}(\mathrm{mmHg})$ & $297 \pm 55$ & $291 \pm 67$ & $319 \pm 50$ & $287 \pm 120$ \\
\hline$\% \mathrm{FVC}$ & $69 \pm 17$ & $78 \pm 28$ & $80 \pm 17$ & $78 \pm 21$ \\
\hline$\%$ DLco & $49 \pm 12$ & $63 \pm 17$ & $56 \pm 11$ & $58 \pm 15$ \\
\hline KL-6 (IU/ml) & $1,927 \pm 617$ & $1,367 \pm 1,239$ & $1,453 \pm 517$ & $817 \pm 328$ \\
\hline
\end{tabular}

CVDs: collagen vascular diseases and related disorders, RA: rheumatoid arthritis, SjS: Sjögren's syndrome, DM: dermatomyositis, MPA: microscopic polyangiitis, C: cellular, F: fibrotic.

deterioration defined by one or more of the following: a) a $10 \%$ decrease in FVC or $>200 \mathrm{ml}$ change, b) a decrease in single-breath DLco of more than $15 \%$ or $>3 \mathrm{ml} / \mathrm{min} / \mathrm{mmHg}$, c) a decrease in resting $\mathrm{PaO}_{2}$ of more than $10 \mathrm{mmHg}$ from the previous level.

\section{Results}

\section{Clinical and histopathological findings}

Among the 33 patients, all had corticosteroid-resistant IP and 9 out of 10 patients with UIP/IPF developed acute exacerbation. They received a low dosage of CsA (50-200 mg/ day, blood trough level: $100-150 \mathrm{ng} / \mathrm{ml}$ ) combined with

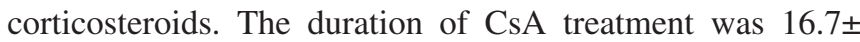
18.7 months (7 days-71 months). Liver dysfunction occurred in one patient $(3.0 \%)$ as an adverse side effect of CsA after 4 months, and improved with discontinuation of CsA treatment. The underlying systemic diseases were: unknown etiology (IPF or IIPs) in 19, and collagen vascular diseases or related disorders (CVDs) in 14. According to the classification of IP, the histopathological pattern and underlying IPrelated systemic diseases were categorized into four groups: 1) usual interstitial pneumonia (UIP)/IPF in 10 patients, 2) non-UIP/IIPs in 9 patients [cellular-nonspecific interstitial pneumonia (NSIP)/IIPs in 3 patients, fibrotic-NSIP/IIPs in 5, $\mathrm{OP} / \mathrm{IIP}$ in one], 3) UIP/CVDs in 4 patients (RA and $\mathrm{SjS}$ in 2 patients each), 4) non-UIP/CVDs in 10 patients [cellularNSIP/CVDs in 7 patients, fibrotic-NSIP/CVDs in 2, and diffuse alveolar damage (DAD)/CVD in one (DM in 7, RA, SjS and MPA in one patient each)], respectively. The mean
$\mathrm{PaO}_{2} / \mathrm{FiO}_{2}$ on initiation of $\mathrm{CsA}$ treatment in the four groups were 287-319 $\mathrm{mmHg}$, and that of \% FVC and \%DLco were 69-80\% and 49-63\%, respectively. Serum KL-6, as an indicator of active pneumonitis, rose from 817 to $1,927 \mathrm{U} / \mathrm{ml}$ (normal values $<500 \mathrm{U} / \mathrm{ml}$ ) in all four groups (15) (Table 1). The mean duration of pretreatment with prednisolone (PSL) prior to administration of CsA ranged from 0.87 to 19.5 months and the dosage of PSL at the initiation of CsA treatment ranged from 26 to $156 \mathrm{mg} /$ day in these four groups (Table 2).

\section{Prognoses and survival rates}

According to the criteria described previously, the responses to treatment with CsA for IP were as follows: improved in 4 patients, unchanged in 12, and deteriorated in 17, respectively. The duration for improvement to start after the CsA treatment was $10.0 \pm 4.8$ days (5 to 15 days).

Prognoses after the treatment with CsA in the four groups were: 1) improved or unchanged in $78 \%$ of patients with non-UIP/IIPs, in $50 \%$ of patients with UIP/CVDs and in $75 \%$ of patients with non-UIP/CVDs, 2) deteriorated in all patients with UIP/IPF, in $22 \%$ of patients with non-UIP/IIPs, in $50 \%$ of patients with UIP/CVDs and in $30 \%$ of patients with non-UIP/CVDs. The prognoses in non-UIP groups were significantly better than those in UIP/IPF (Table 3, Fig. 1).

Furthermore, the relationship between detailed histopathological patterns and prognoses after CsA treatment in the eight groups were: 1) improved or unchanged in all patients of cellular NSIP/IIPs, in $86 \%$ of cellular NSIP/CVDs, in $60 \%$ of fibrotic NSIP/IIPs, in $50 \%$ of fibrotic NSIP/CVDs, in 
Cyclosporin Treatment in Interstitial Pneumonia

Table 2. Pretreatment with Prednisolone before Administration of CsA

\begin{tabular}{lccc}
\hline $\begin{array}{c}\text { Histological pattern/underlying } \\
\text { sistemic diseases }\end{array}$ & $\begin{array}{c}\text { Number } \\
\text { of patients }\end{array}$ & $\begin{array}{c}\text { Duration of pretreatment } \\
\text { with PSL (months) }\end{array}$ & $\begin{array}{c}\text { PSL dose at the initiation } \\
\text { of CsA (mg/day) }\end{array}$ \\
\hline UIP/IPF & 10 & $\begin{array}{c}0.87 \pm 1.2 \\
(0-3.5)\end{array}$ & $156 \pm 385$ \\
Non-UIP/IIPs & 9 & $9.4 \pm 14.4$ & $33 \pm 15$ \\
UIP/CVDs & 4 & $\begin{array}{c}(0.1-39.6) \\
19.5 \pm 28\end{array}$ & $26 \pm 7.5$ \\
& & $(1-60)$ & $40 \pm 20$ \\
Non-UIP/CVDs & 10 & $12.6 \pm 28.2$ & \\
& & $(0.1-91.2)$ & \\
\hline
\end{tabular}

UIP: usual interstitial pneumonia, IPF: idiopathic pulmonary fibrosis, IIP: idiopathic interstitial pneumonia, CVD: collagen vascular disease, PSL: prednisolone.

Table 3. Histological Pattern/underlying Systemic Diseases and Prognoses in Four Subgroups with IP after Treatment with CsA

\begin{tabular}{|c|c|c|c|c|c|}
\hline $\begin{array}{l}\text { Histological pattern/underlying } \\
\text { systemic diseases }\end{array}$ & Number of patients & Improved & Unchanged & Deteriorated & \\
\hline UIP/IPF & 10 & $0(0 \%)$ & $0(0 \%)$ & $10(100 \%)$ & \multirow{4}{*}{$*$} \\
\hline Non-UIP/IIPs & 9 & $1(11 \%)$ & $6(67 \%)$ & $2(22 \%) *$ & \\
\hline UIP/CVDs & 4 & $0(0 \%)$ & $2(50 \%)$ & $2(50 \%)$ & \\
\hline Non-UIP/CVDs & 10 & $3(30 \%)$ & $4(40 \%)$ & $3(30 \%)$ & \\
\hline
\end{tabular}

$*: \mathrm{p}<0.05$

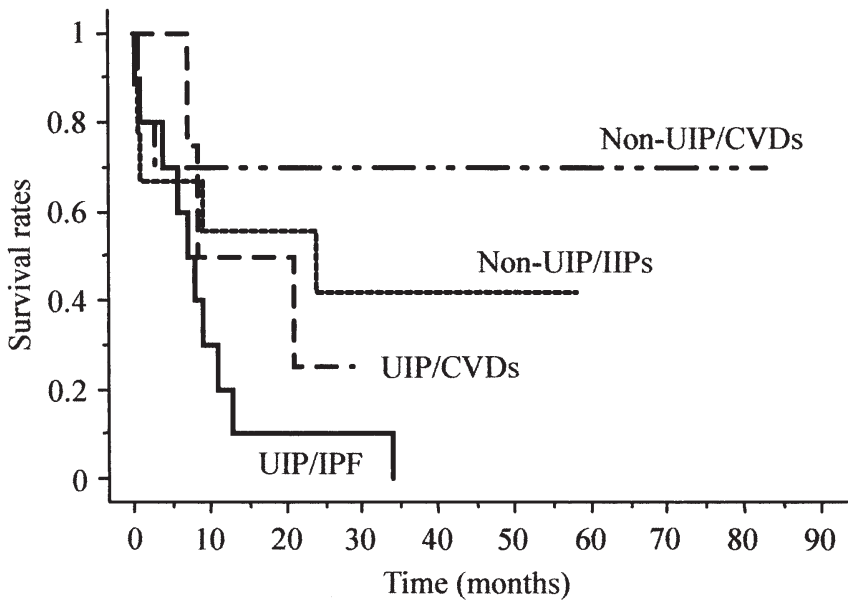

Figure 1. Comparison of survival rates after treatment with CsA in four subgroups with IP (Kaplan and Meier method). The survival was significantly worse in the UIP/IPF subgroup than in the non-UIP/CVDs subgroup $(\mathbf{p}<0.05)$.

$50 \%$ of UIP/CVDs and in one patient of OP/IIP, 2) deteriorated in $0 \%, 14 \%, 40 \%, 50 \%$, and $50 \%$ of patients with the above-mentioned patterns and underlying diseases, in all patients with UIP/IPF, and in one patient with DAD/CVD. Interestingly, the individuals who showed improvement to
CsA belonged only to the cellular NSIP groups among these eight categories. The prognoses after CsA treatment were well correlated with histopathological patterns. CellularNSIP and OP showed better prognoses than fibrotic-NSIP, UIP or DAD. In addition, CVDs had more favorable prognoses than IIPs when compared with the same corresponding histopathological patterns (Table 4, Fig. 2).

Comparison of the survival rates after the onset of acute exacerbation in patients with UIP/IPF showed that the mean survival time in the CsA-treated group ( 9 patients) was 9.9 months, while that of the non-CsA-treated group (35 patients) was 1.7 months. Furthermore, 3 out of 9 patients in the CsA-treated group survived for more than eleven months. Statistically, the prognoses in the CsA-treated group were significantly better than in those without CsA treatment in acute exacerbation of UIP/IPF (Table 5, Fig. 3).

\section{Discussion}

The efficacy of CsA in treating interstitial pneumonia has been studied in patients with collagen vascular diseases. For example, Nawata et al (2) reported that CsA was effective in all 5 patients with corticosteroid-resistant IP with DM, and that their one-year survival rate reached $80 \%$. Nagasaka et al (16) recently reported that primary use of CsA with corticosteroids rescued 9 of 13 acute IP patients (69\%) with DM. In contrast, initial treatment with corticosteroid alone 
Homma et al

Table 4. Histological Pattern/underlying Systemic Diseases and Prognoses in Eight Subgroups with IP after Treatment with CsA

\begin{tabular}{|c|c|c|c|c|}
\hline \multicolumn{2}{|c|}{$\begin{array}{l}\text { Histological pattern/underlying } \\
\text { systemic diseases }\end{array}$} & Improved & Unchanged & Deteriorated \\
\hline UIP $(n=14)$ & $\begin{array}{l}\text { IPF (10) } \\
\text { CVD (4) } \\
\text { (RA: 2, SjS: 2) }\end{array}$ & $\begin{array}{l}0(0 \%) \\
0(0 \%)\end{array}$ & $\begin{array}{l}0(0 \%) \\
2(50 \%)\end{array}$ & $\begin{array}{c}10(100 \%) \\
2(50 \%)\end{array}$ \\
\hline C-NSIP $(n=10)$ & $\begin{array}{l}\text { IIP (3) } \\
\text { CVD (7) } \\
\text { (DM: 5, RA: 1, MPA: 1) }\end{array}$ & $\begin{array}{l}1(33 \%) \\
3(43 \%)\end{array}$ & $\begin{array}{l}2(67 \%) \\
3(43 \%)\end{array}$ & $\begin{array}{l}0(0 \%) \\
1(14 \%)\end{array}$ \\
\hline F-NSIP $(n=7)$ & $\begin{array}{l}\text { IIP (5) } \\
\text { CVD (2) } \\
\text { (DM: } 1, \mathrm{SjS}: 1)\end{array}$ & $\begin{array}{l}0(0 \%) \\
0(0 \%)\end{array}$ & $\begin{array}{l}3(60 \%) \\
1(50 \%)\end{array}$ & $\begin{array}{l}2(40 \%) \\
1(50 \%)\end{array}$ \\
\hline OP $(n=1)$ & IIP (1) & $0(0 \%)$ & $1(100 \%)$ & $0(0 \%)$ \\
\hline $\operatorname{DAD}(n=1)$ & $\begin{array}{l}\text { CVD (1) } \\
\text { (AMDM: 1) }\end{array}$ & $0(0 \%)$ & $0(0 \%)$ & $1(100 \%)$ \\
\hline
\end{tabular}

AMDM: amyopathic dermatomyositis.

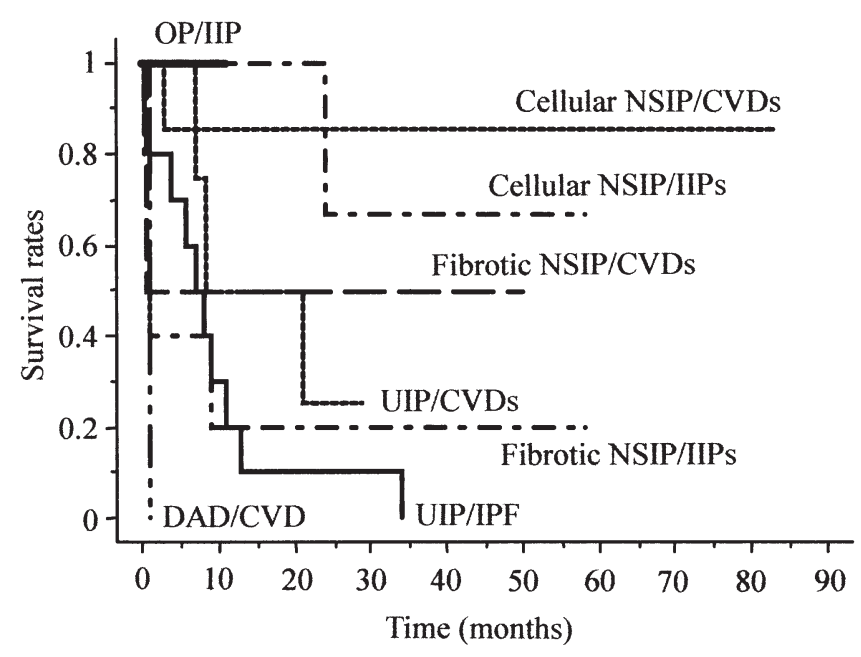

Figure 2. Comparison of survival rates after treatment with CsA in eight subgroups with IP (Kaplan and Meier method).

rescued only 8 of 25 patients (32\%). Recently, we also reported a case of corticosteroid-resistant refractory cellular NSIP associated with DM who had been successfully treated with CsA (17).

However, the mechanisms by which CsA could prevent the progression of corticosteroid-resistant IP in DM/PM, and the etiology itself of IP in DM/PM remains to be determined. CsA acts as an immunomodulator, predominantly suppressing Th1 lymphocyte function and proliferation, suggesting that $\mathrm{T}$ cells play important roles in the pathogenesis of corticosteroid-resistant IP in DM/PM. Furthermore, recent studies have shown that the action of CsA in blocking signal pathways which are important for $\mathrm{T}$ cell activation, is different from that of corticosteroids. CsA inhibits the action of calcineurin, which eventually render a complete blockade of translocation of the cytosolic component of the NF-AT transcription factor. Consequently, activation of certain genes which are regulated by NF-AT, such as IL-4 and IL-2, would be suppressed (4). Moreover, it has been reported that CsA could inhibit proliferation of activated $\mathrm{T}$ cells on which corticosteroids have little effect (18). Therefore, corticosteroid-resistant IP that is likely caused by activated $\mathrm{T}$ cells could be ameliorated by CsA, but not by corticosteroid.

In the present study, the mean survival time after the introduction of CsA treatment in seven patients with corticosteroid-resistant IP associated with DM was 22.2 months, comprising improved or unchanged in 4 patients with cellular-NSIP, and deteriorated condition in a patient each with cellular-NSIP, fibrotic-NSIP, and DAD, respectively. The prognoses after initiating CsA treatment in our series of patients as a whole are very similar to those reported in the previous studies (19-22). Cellular-NSIP and OP showed better prognoses than fibrotic-NSIP, UIP or DAD. In addition, CVDs had better prognoses than IIPs when compared with the same corresponding histopathological patterns. These results might be explained by the efficacy of CsA on IP associated with CVDs but not on IIPs.

The effectiveness of CsA on IPF is controversial. For example, Alton et al (23) reported a poor response to CsA after a long-term use, although the initial response was favorable: the mean survival time in CsA-treated patients was doubled from 2.5 to 5 months, in comparison with the controls closely matched for disease severity. On the contrary, a study by Moolman et al (24) showed that 3 of 5 patients responded to CsA and corticosteroid with an improvement in dyspnea and an increase in vital capacity 6 months after treatment. Regarding acute exacerbation of IPF, Inase et al (25) 
Cyclosporin Treatment in Interstitial Pneumonia

Table 5. Comparison of Prognoses between CsA-treated Group and Non-CsA-treated Group in Patients with Acute Exacerbation of UIP/IPF

\begin{tabular}{|c|c|c|c|c|c|}
\hline Document & $\begin{array}{l}\text { Number } \\
\text { of patients }\end{array}$ & Alive & $\begin{array}{l}\text { (Duration after } \\
\text { the onset of } \mathrm{AE} \text { ) }\end{array}$ & Died & $\begin{array}{l}\text { (Duration after } \\
\text { the onset of } \mathrm{AE} \text { ) }\end{array}$ \\
\hline \multicolumn{6}{|l|}{ With CsA therapy } \\
\hline The present study & 9 & $0(0 \%)$ & & $9(100 \%)$ & $\frac{9.9 \pm 10.3 \mathrm{mo}}{(0.34-35 \mathrm{mo})}$ \\
\hline Inase et al & 7 & $4(57 \%)$ & $\begin{array}{c}38.8 \pm 22.1 \mathrm{mo} \\
(14-64 \mathrm{mo})\end{array}$ & $3(43 \%)$ & $\begin{array}{l}10.3 \pm 15.3 \mathrm{mo} \\
(0.93-28 \mathrm{mo})\end{array}$ \\
\hline \multicolumn{6}{|l|}{ Without CsA therapy } \\
\hline Yoshimura et al & 35 & $0(0 \%)$ & & $35(100 \%)$ & $\frac{1.7 \pm 3.2 \mathrm{mo}}{(0.1-19 \mathrm{mo})}$ \\
\hline Inase et al & 6 & $0(0 \%)$ & & $6(100 \%)$ & $\begin{array}{c}4.2 \pm 5.7 \mathrm{mo} \\
(0.5-15 \mathrm{mo})\end{array}$ \\
\hline Ambrosini et al & 5 & $1(20 \%)$ & $18 \mathrm{mo}$ & $4(80 \%)$ & $\begin{array}{c}0.44 \pm 0.37 \mathrm{mo} \\
(0.23-1 \mathrm{mo})\end{array}$ \\
\hline
\end{tabular}

AE: acute exacerbation.

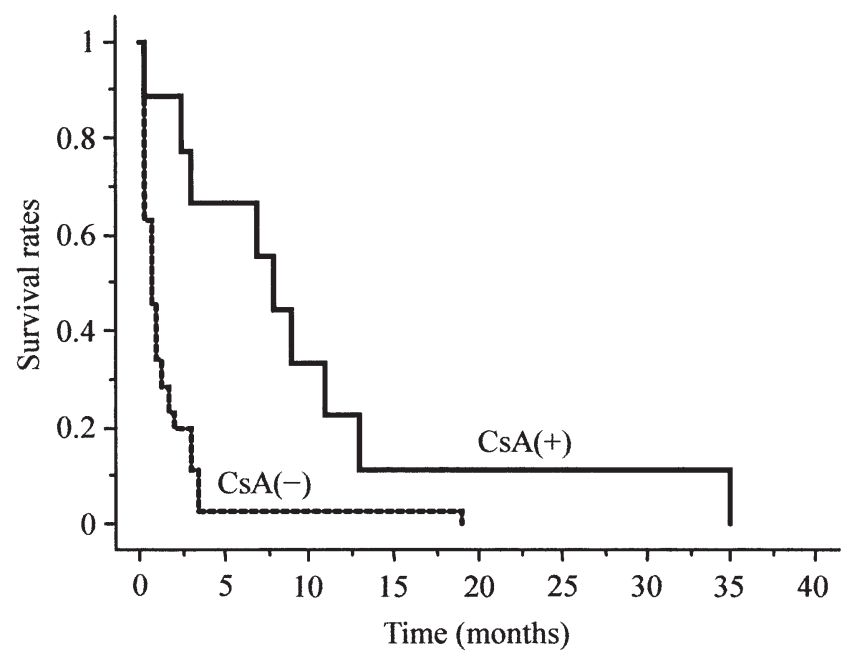

Figure 3. Comparison of survival rates between the CsAtreated group (solid line, $n=9$ ) and the non-CsA-treated group (dotted line, $n=35$ ) of patients with UIP/IPF who developed acute exacerbation (Kaplan and Meier method). The survival was significantly worse in the non-CsA-treated group than in the CsA-treated group $(\mathbf{p}<0.01)$.

described their experience in treating 7 patients who received CsA in comparison with the outcomes of 6 IPF patients who did not have CsA. Among these 7 patients treated with CsA, 4 patients had survived for 14, 28, 64 or 49 months, respectively. In contrast, all 6 patients receiving no CsA died within 15 months from the onset of acute exacerbation. Recently, Ambrosini et al (26) also reported that 4 out of 5 patients who did not receive CsA died within one month after the onset of acute exacerbation. The present study has demonstrated that the CsA-treated group had a significantly better prognosis than that without CsA treatment in terms of recovering from acute exacerbation of UIP/IPF. Among the 9 patients treated with CsA, 6 patients had survived for 7, 8 , $9,11,13$ and 35 months after acute exacerbation. In contrast, 34 out of 35 patients who did not receive CsA died within 4 months after the onset of acute exacerbation as was previously reported by our group (11).

In conclusion, CsA combined with corticosteroids may be an efficacious treatment for corticosteroid-resistant IP and for acute exacerbation of IPF. Further randomized controlled studies are required to determine the long-term effectiveness of the treatment.

Acknowledgements: This work was supported by a Grant-In-Aid for Scientific Research from the Japanese Ministry of Education, Sciences, Sports and Culture (S.H.)

\section{References}

1) Gruhn WB, Diaz-Buxo JA. Cyclosporine treatment of steroid resistant interstitial pneumonitis associated with dermatomyositis/polymyositis. J Rheumatol 14: 1045-1047, 1987.

2) Nawata $Y$, Kurasawa K, Takabayashi K, et al. Corticosteroid resistant interstitial pneumonitis in dermatomyositis/polymyositis: prediction and treatment with cyclosporine. J Rheumatol 26: 1527-1533, 1999.

3) Kurasawa K, Nawata Y, Takabayashi K, et al. Activation of pulmonary $\mathrm{T}$ cells in corticosteroid-resistant and -sensitive interstitial pneumonitis in dermatomyositis/polymyositis. Clin Exp Immunol 129: 541-548, 2002.

4) Ho S, Clipstone N, Timmermann L, et al. The mechanism of action of cyclosporin A and FK506. Clin Immunol Immunopathol 80: S40-S45, 1996.

5) Jonat C, Rahmsdorf HJ, Park KK, et al. Antitumor promotion and antiinflammation: down-modulation of AP-1 (Fos/Jun) activity by glucocorticoid hormone. Cell 62: 1189-1204, 1990.

6) American Thoracic Society/European Respiratory Society International Multidisciplinary Consensus Classification of the Idiopathic Interstitial Pneumonias. Am J Respir Crit Care Med 165: 277-304, 2002. 


\section{HoMma et al}

7) Vitali C, Bombardieri S, Moutsopoulos HM, et al. Preliminary criteria for the classification of Sjögren's syndrome. Results of a prospective concerted action supported by the European Community. Arthritis Rheum 36: 340-347, 1993.

8) Bohan A, Peter JB. Polymyositis and dermatomyositis. N Engl J Med 292: 344-347, 403-407, 1975.

9) Jennette JC, Falk RJ, Andrassy K, et al. Nomenclature of systemic vasculitides: proposal of an international consensus conference. Arthritis Rheum 37: 187-192, 1994.

10) Arnett FC, Edworthy SM, Bloch DA, et al. The American Rheumatism Association 1987 revised criteria for the classification of rheumatoid arthritis. Arthritis Rheum 31: 315-324, 1988.

11) Yoshimura $K$, Nakatani $T$, Nakamori $Y$, et al. Acute exacerbation in idiopathic interstitial pneumonia. Nihon Kyobu Shikkan Gakkai Zasshi 22: 1012-1020, 1984 (Abstract in English).

12) Tamura M. Diagnostic criteria of idiopathic interstitial pneumonia. Annual report on the study of interstitial lung disease. Grant-in-Aid from the Ministry of Health and Welfare in Japan 4-6, 1980 (in Japanese).

13) Akira M, Hamada H, Sakatani M, Kobayashi C, Nishioka M, Yamamoto S. CT findings during accelerated deterioration in patients with idiopathic pulmonary fibrosis. AJR 168: 79-83, 1997.

14) American Thoracic Society. International consensus statement. Idiopathic pulmonary fibrosis: diagnosis and treatment. Am J Respir Crit Care Med 161: 646-664, 2000.

15) Kohno N, Kyoizumi S, Awaya Y, Fukuhara H, Yamakido M, Akiyama M. New serum indicator of interstitial pneumonitis activity. Chest 96: 68-73, 1989.

16) Nagasaka K, Harigai M, Tateishi M, et al. Efficacy of combination treatment with cyclosporin A and corticosteroids for acute interstitial pneumonitis associated with dermatomyositis. Mod Rheumatol 13: 231-238, 2003.

17) Sakamoto S, Homma S, Kawabata M, et al. A case of corticosteroidresistant nonspecific interstitial pneumonia associated with dermato- myositis successfully treated with cyclosporin A. Nihon Kokyuki Gakkai Zasshi 43: 171-178, 2005 (Abstract in English).

18) Brinkmann V, Kristofic C. Regulation by corticosteroids of Th1 and Th2 cytokine production in human $\mathrm{CD}^{+}$effector $\mathrm{T}$ cells generated from $\mathrm{CD}^{2} 4 \mathrm{RO}^{-}$and $\mathrm{CD}^{2} 4 \mathrm{RO}^{+}$subsets. J Immunol 155: 3322-3328, 1995.

19) Daniil ZD, Gilchrist FC, Nicholson AG, et al. A histologic pattern of nonspecific interstitial pneumonia is associated with a better prognosis than usual interstitial pneumonia in patients with cryptogenic fibrosing alveolitis. Am J Respir Crit Care Med 160: 899-905, 1999.

20) Nicholson AG, Colby TV, du Bois RM, Hansell DM, Wells AU. The prognostic significance of the histologic pattern of interstitial pneumonia in patients presenting with the clinical entitiy of cryptogenic fibrosing alveolitis. Am J Respir Crit Care Med 162: 2213-2217, 2000.

21) Travis WD, Matsui K, Moss J, Ferrans VJ. Idiopathic nonspecific interstitial pneumonia: prognostic significance of cellular and fibrosing patterns. Am J Surg Pathol 24: 19-33, 2000.

22) Nagai $S$, Kitaichi $M$, Itoh $H$, Nishimura $K$, Izumi $T$, Colby $T V$. Idiopathic nonspecific interstitial pneumonia/fibrosis: comparison with idiopathic pulmonary fibrosis and BOOP. Eur Respir J 12: 1010-1019, 1998 (Erratum in: Eur Respir J 13: 711, 1999).

23) Alton EW, Johnson M, Turner-Warwick M. Advanced cryptogenic fibrosing alveolitis: preliminary report on treatment with cyclosporin A. Respir Med 83: 277-279, 1989.

24) Moolman JA, Bardin PG, Rossouw DJ, Joubert JR. Cyclosporin as a treatment for interstitial lung disease of unknown aetiology. Thorax 46: 592-595, 1991.

25) Inase N, Sawada M, Ohtani $Y$, et al. Cyclosporin A followed by the treatment of acute exacerbation of idiopathic pulmonary fibrosis with corticosteroid. Intern Med 42: 565-570, 2003.

26) Ambrosini V, Cancellieri A, Chilosi M, et al. Acute exacerbation of idiopathic pulmonary fibrosis: report of a series. Eur Respir J 22: 821826, 2003. 